\section{Increased adolescent mortality in the year after a psychiatric admission}

\section{QUESTION}

Question: What are the rates of admissions for psychiatric conditions in adolescents in England, and what is the subsequent 1-year mortality?

Population: Adolescents aged between 10 and 19 years of age.

Setting: Psychiatric admissions to National Health Service hospitals between 1998 and 2004 from Hospital Episode Statistics (HES).

Assessment: The total number of admissions for each age group was determined from HES data. Admission rates for each age group were calculated separately for males and females where numerators were those admitted in each agesex group and denominators were the relevant total from the English population. Age- and sex-specific admission rates were determined overall and for specific psychiatric disorders according to International Classification of Diseases (ICD-10) groupings. Age- and gender-specific admission rates were determined with and without including substance abuse.

Outcomes: Mortalities: All admission records were linked to death certificate data, and age- and sex-specific mortalities were determined for each diagnostic group by year of age at admission. Standardised Mortality Ratios (SMRs) were then calculated to assess the differences in observed and expected deaths where expected deaths were estimated from general population data in England.

\section{METHODS}

Design: Cross-sectional study.

\section{MAIN RESULTS}

Between 1998 and 2004, there were 57783 adolescent psychiatric admissions for schizophrenia; affective, neurotic, eating, developmental, personality or behavioural disorders; or alcohol and substance abuse. More than $50 \%$ of admissions overall were due to alcohol and substance abuse. Total hospital admission rates were presented graphically. When admissions due to substance abuse were included, the rate per 1000 seemed highest in those aged 19 although there was little difference between this group and those aged 15 years. The highest rates were seen in males aged 18 and 19 and females aged 14 and 15 . When substance abuse was excluded, admissions per 1000 rose from age 10 to 19 . The highest rates appeared to be in males and females aged 18 and 19.

Schizophrenia was the second most common reason for admission following alcohol and substance misuse. Admissions were more common in males and peaked at 19 years ( 1.5 per 1000 people). Twenty-four per cent of all psychiatric admissions below age 19 were due to schizophrenia not including alcohol and substance abuse. Admissions were generally more common in females for affective disorders, neurotic disorders and eating disorders which peaked in females at 15 years. Admissions for neurodevelopmental disorders declined steadily from 11 to 19 years with a male excess.

A total of 120 adolescents ( $0.23 \%$ of males, $0.18 \%$ of females) died in the year following admission. Cause of death data were not available. The overall SMR for the population across all disorders was 642 (95\% CI 532 to 767), a 542\%increase in expected deaths in adolescents following an admission compared with the general population. The SMR was higher for females aged 10-19 than for males (933 vs 518). When analysed by age group, SMRs were highest in the younger and older age groups. When ages were combined, SMRs were greatest in adolescents with developmental disorders, eating disorders and affective disorders (3017 vs 1103 vs 940, respectively) although CIs were wide because of small numbers who died in each subgroup.

\section{CONCLUSIONS}

Admissions for psychiatric disorders increase sharply for teenagers with increasing age. The death rate within a year of discharge is six times higher in this group than in the general population of the same age.

\section{ABSTRACTED FROM}

James A, Clacey J, Seagroatt V, et al. Adolescent inpatient psychiatric admission rates and subsequent one-year mortality in England: 1998-2004. J Child Psychol Psychiatry 2010;51:1395-404.

Correspondence to: Anthony James, Highfield Adolescent Unit, Warneford Hospital, Oxford OX3 7JX, UK; anthony.james@psych.ox.ac.uk

Source of funding: NIHR Centre for Research Capacity Development.

- Notes are available online only at www.ebmh.bmj.com
$\mathrm{T}$ he study by James and colleagues addresses the important issue of psychiatric inpatient admission rates for the adolescent population. They used the large UK data source of the Hospital Episode Statistics - information that is recorded on every public hospital admission in England - and link this to death certificate data to investigate admission rates and mortalities during the year following discharge. To date, in the UK, the use of these important large national databases has been restricted to adult psychiatric populations. ${ }^{1}$ They report a logarithmic increase in admission rates for psychiatric illness from the age of 10 up to the age of 19. Admission rates are approaching those reported for adult psychiatric populations at the age of 19 (2.2 per 1000). They also report significantly increased Standardised Mortality Ratios in the year post discharge from hospital, both generally and especially for particular disorders (developmental disorders and eating disorders), although the overall number of deaths was relatively low (120). The authors acknowledge limitations of the lack of data on private admissions and that the data relate to 1998-2004 and are therefore not as contemporary as they would like. The study raises a number of important issues: although, as the authors point out, inpatient admission is only a proxy for morbidity, it does add further support to the high level of psychiatric need in adolescent years, a time of increasing incidence for most major psychiatric disorders in the context of specific developmental challenges. ${ }^{2}$ Allied to this, the reported-raised mortalities in all psychiatric disorders are a major public health issue in young people. Finally, the number of child psychiatric admissions to adult psychiatric beds is another worrying finding and one that needs to be addressed by policy makers and service planners.

\section{Andrew Thompson}

Orygen Youth Health and Orygen Research Centre, Centre for Youth Mental Health, University of Melbourne, Melbourne, Victoria, Australia

\section{Competing interests None.}

\section{REFERENCES}

1. Thompson A, Shaw M, Harrison G, et al. Patterns of hospital admission for adult psychiatric illness in England: analysis of Hospital Episode Statistics data. Br J Psychiatry 2004;185:334-41.

2. McGorry PD. The specialist youth mental health model: strengthening the weakest link in the public mental health system. Med J Aust 2007;187 (7 Suppl):S53-6. 